\title{
Assessing meso- and microplastic pollution in the Ligurian and Tyrrhenian Seas
}

\author{
Jessica Caldwell ${ }^{\mathrm{a}}$, Alke Petri-Fink ${ }^{\mathrm{a}}$, Barbara Rothen-Rutishauser ${ }^{\mathrm{a}}$, Roman Lehner ${ }^{\mathrm{a}, \mathrm{b}, *}$ \\ ${ }^{a}$ Adolphe Merkle Institute, Université de Fribourg, Chemin de Verdiers 4, 1700 Fribourg, Switzerland \\ ${ }^{\mathrm{b}}$ Sail and Explore Association, Kirchweg 42, 8755 Ennenda, Switzerland
}

\section{A R T I C L E I N F O}

\section{Keywords:}

Mesoplastic

Microplastic

Environmental pollution

Fourier transform infrared spectroscopy (FT-IR)

Mediterranean

Tyrrhenian Sea

Ligurian Sea

\begin{abstract}
A B S T R A C T
As the production of plastic products continues to increase, determining the fate of plastic waste in the environment is of high importance. Densely populated areas, such as Mediterranean coastlines, represent locations of high pollution risk for surrounding environments. Thus, this study aims to assess the abundance, size, and composition of floating meso- and microplastics collected during four weeks in 2018 in the Ligurian and Tyrrhenian Seas. The results show average meso- and microplastic particle concentrations of 28,376 $\pm 28,917$ particles $\mathrm{km}^{-2}$, and an average mass of $268.61 \pm 421.18 \mathrm{~g} \mathrm{~km}^{-2}$. The particle shape ratio was $65 \%$ fragments, $19 \%$ films, $10 \%$ lines, $4 \%$ foams, and $2 \%$ pellets. Microplastic particles comprised $65 \%$ of the sample. Analysis with attenuated total reflection Fourier transform infrared spectroscopy showed predominant polymer types included polyethylene, polypropylene, polystyrene, and polyamide. These data are an important starting point for long-term monitoring of plastic pollution levels within this region.
\end{abstract}

\section{Introduction}

Plastics have a wide range of structural and functional properties that are exploited by multiple industries ranging from packaging to construction to automotive (PlasticsEurope, 2018). In 2017, worldwide production of plastics was 348 million tons, which was an increase of 13 million tons from the number reported for 2016 (PlasticsEurope, 2017, 2018). This continuous growth of the plastics industry also results in an increase in the amount of plastic waste produced worldwide. In 2010, researchers presented numbers in the range of 4.8 to 12.7 million tons of mismanaged plastic waste that was generated in 192 coastal countries and then discarded into the oceans (Jambeck et al., 2015). The Pacific Garbage Patch, a highly polluted ocean region between Hawaii and California, had values as high as 21 thousand tons of plastic present in 2014 (Eriksen et al., 2014). More recent studies in this region, however, give values significantly higher; with a study published in 2018 giving an estimate of 79 thousand tons of plastic present (Lebreton et al., 2018). These ever-increasing pollution levels highlight the importance of monitoring plastics in natural environments.

Studies have observed that the combination of mechanical force from phenomena such as ocean waves and UV radiation from the sun can cause the breakdown of common macro-sized plastic waste into micro-sized ( $5 \mathrm{~mm}-1 \mu \mathrm{m}$ ) particles, known as microplastics (Wright and Kelly, 2017). Thus, weathering phenomena increases the overall plastic particle concentration within the environment and the potential risk of interaction with and ingestion of these particles by various species. In a study conducted on species of pelagic fish collected from Mediterranean waters, $58 \%$ of the fish had ingested microplastic particles, and skin biopsies from fin whales also showed traces of phthalates commonly used in plastic products (Güvena et al., 2017; Fossi et al., 2016). Effects of ingesting these particles can range from pro-inflammatory response to decreased feeding and growth rates and ultimately to starvation for a wide variety of aquatic species (von Moos et al., 2012; Connors and Smith, 1982; Watts et al., 2015; Moore, 2008). These effects can be further exacerbated by the adsorption of organic and heavy metal pollutants to the plastic particles, which can then desorb upon their ingestion, or by the leaching of common additives like phthalates (Fossi et al., 2016; Brennecke et al., 2016; Rochman et al., 2013).

These microplastic particles have already been detected in environments ranging from beach sediment and surface waters all around the globe to more remote locations such as deep sea sediments and arctic waters (Browne et al., 2011; Lusher et al., 2015; Van Cauwenberghe et al., 2013). The Mediterranean Sea is predicted to be highly impacted by plastic particle pollution (van Sebille et al., 2015). This high particle concentration is likely the result of the population density within the area; a $10 \mathrm{~km}$ coastal strip along the Mediterranean is estimated to be home to approximately 100 million people (Center for International Earth Science Information Network (CIESIN), 2012).

\footnotetext{
* Corresponding author.

E-mail address: roman.lehner@unifr.ch (R. Lehner).
} 
The nearly 200 million tourists which visit the region within the year also have a significant impact on the waste generated in the region, with the amount of marine litter alone being increased by $40 \%$ during the summer season (Galgani, 2014).

Current estimates for the Mediterranean Sea indicate approximately $7 \%$ of the global microplastics present in the marine environment are within the region, and thus it is the world's 6th largest accumulation zone for marine litter; as much as 1.25 microplastic fragments $\mathrm{km}^{-2}$ are present within its waters and another 10,000 per $\mathrm{km}^{-2}$ is present in the sediment on the sea's floor (Galgani et al., 2000; Suaria et al., 2016). Multiple studies, a select few of which are shown in Table 1, have been conducted in an effort to begin to accurately quantify the amount of plastic particles present within various sub-regions of the Mediterranean. Studies such as these will be an important tool for monitoring and assessing the increasing pollution levels. Therefore, the aim of this work is to further evaluate the abundance, size and composition of floating plastics, including both mesoplastics $(20 \mathrm{~cm}$ to $5 \mathrm{~mm}$ ) and microplastics, in the Ligurian and the Tyrrhenian Seas. These results will help to provide insight into the high variability of particle concentrations present environmentally, and aid in the long-term monitoring of the pollution levels within the region.

\section{Materials and methods}

\subsection{Sample collection}

34 surface samples were collected in the Ligurian and Tyrrhenian Seas (Fig. 1) between May 19th and June 15th, 2018. Samples were collected using a manta trawl with $330 \mu \mathrm{m}$ mesh size $(16 \times 61 \mathrm{~cm}$ rectangular frame opening) that was towed on the water surface for $30-60 \mathrm{~min}$ at $1.5-3$ knots on either starboard or port side. A spinnaker pole was used to position the trawl outside the wake of the vessel and to avoid loss of samples due to downwelling of debris. The outer side of the net was rinsed with fresh water to ensure that the entire sample was washed into the collecting bag fixed at the end of the net. This sample was emptied over a metal sieve with a mesh size of $300 \mu \mathrm{m}$.

\subsection{Sample preparation and visual identification}

Plastic particles which could be visually detected in the metal sieve were collected using laboratory tweezers. In addition, saltwater, filtered through a sieve with a mesh size of $0.1 \mathrm{~mm}$, was added into sample containers, which separated the plastic particles from the preserved natural material according to their density. Floating plastic particles at the surface were removed for further studies. Sorting of the material was conducted using a dissecting stereo microscope (Leica) and laboratory tweezers to hand pick the plastics, which were then transferred to $50 \mathrm{ml}$ Falcon tubes. All samples were double checked by two crew members to ensure detection of all the particles.

\subsection{Classification}

Particles visually identified as plastics were returned to the lab for further classification. The sampled particles were placed on a grid composed of $5 \mathrm{~mm}$ by $5 \mathrm{~mm}$ squares to differentiate mesoplastic particles from microplastic particles. Sorted particles were photographed with a Canon EOS 70D.

Five categories were selected for further particle sorting based on their shape and physical properties: fragments, foams, films, pellets, and lines. Upon completion of particle sorting, particles were covered and allowed to dry at room temperature. The mass of the dried particles was obtained using an AG204 Delta Range balance.

From the five shape categories, 140 total representative particles were randomly selected at a ratio similar to their occurrence in the full sample (Fig. 2). However, in order to ensure a single particle was not 


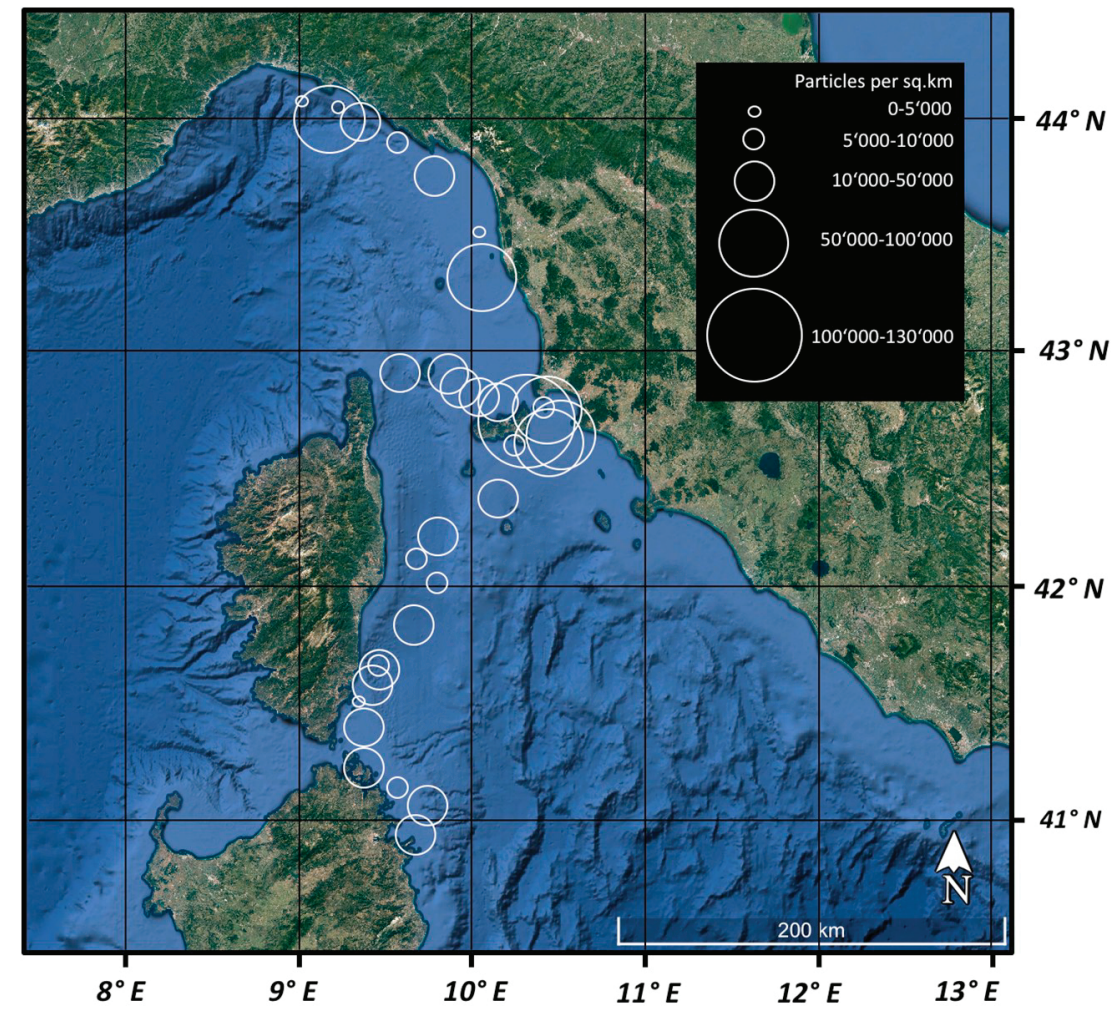

Fig. 1. Map of the study area showing the locations of all transects and the particle concentration (circles) expressed as number of particles per square kilometer Circle size reflects meso- and microplastic concentration (according to the legend shown in the top right corner) of each transect.

\section{Particle Size}

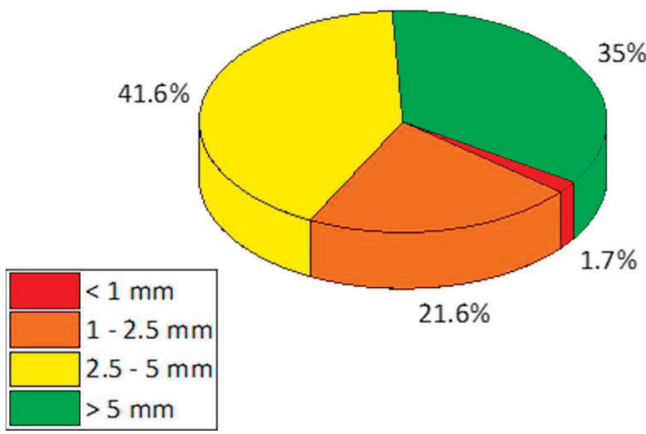

Fig. 2. The ratios for the size of all plastic particles present in the samples.

used to represent an entire shape category, a minimum of 10 representative particles were selected.

\subsection{Fourier transform infrared spectroscopy}

Attenuated total reflection Fourier transform infrared spectroscopy (ATR FT-IR) was utilized to identify the composition of the 140 representative particles (78 fragments, 24 films, 17 lines, 11 pellets, 10 foams). A Perkin Elmer Spectrum 65 FT-IR Spectrometer equipped with a Perkin Elmer universal attenuated total reflection (UATR) accessory and pressure arm was used to collect a combination of 10 spectra from $4000 \mathrm{~cm}^{-1}$ to $650 \mathrm{~cm}^{-1}$ for each particle. The resulting spectra were analyzed with the accompanying Perkin Elmer software and compared to the pristine polymer FT-IR spectra found in the Fluka library.

\subsection{Data processing}

The sample area was calculated using the Global Positioning System data of the length of sea surface trawled multiplied with the width of the trawl. Particle concentrations collected within the net were divided by this distance value and reported as number of particles per square kilometer (particles $\mathrm{km}^{-2}$ ) and mass per square kilometer $\left(\mathrm{g} \mathrm{km}^{-2}\right)$. All tabulation was done using Microsoft Office 365 Excel workbooks and chart creation utilized Origin 2016 software. Final particle size analysis, utilized to obtain the height and width of all particles, was conducted utilizing Fiji (ImageJ version 1.52).

\section{Results}

In each of the 34 samples collected plastic particles were present. Microplastic particles accounted for $65 \%$ of the sample, with the majority of the microplastic particles (41\%) being within the range of 2.5-5 mm (Fig. 2). The plastic concentration ranged from 1,009 to 122,817 particles $\mathrm{km}^{-2}$ depending upon the sample location. The average total particle concentration is 28,376 particles $\mathrm{km}^{-2}$. An average abundance of 9,156 mesoplastic particles (larger than $5 \mathrm{~mm}$ ) $\mathrm{km}^{-2}$ and 19,220 microplastic particles $\mathrm{km}^{-2}$ was calculated for the samples. The mesoplastic particle concentration ranged from 0 particles $\mathrm{km}^{-2}$ to 38,082 particles $\mathrm{km}^{-2}$ while microplastic particle concentrations ranged from 505 particles $\mathrm{km}^{-2}$ to 93,983 particles $\mathrm{km}^{-2}$.

Particles were then further sorted according to their shape. The ratio of the particle shape for each size category can be seen in Fig. 3.

Mass balancing the dried particles revealed an average mass of $268.61 \pm 421.18 \mathrm{~g} \mathrm{~km}^{-2}$ of plastic particles, with the mass ranging from 0.45 to $1,999.79 \mathrm{~g} \mathrm{~km}^{-2}$. Fragments account for the majority of the total mass at approximately $69 \%$ (Fig. 4). This result is in good agreement with the results obtained from particle counts, which show fragments account for approximately $65 \%$ of the total sample (Fig. 5).

The overall ratio for particle shape according to count number, seen 


\section{Microplastics}

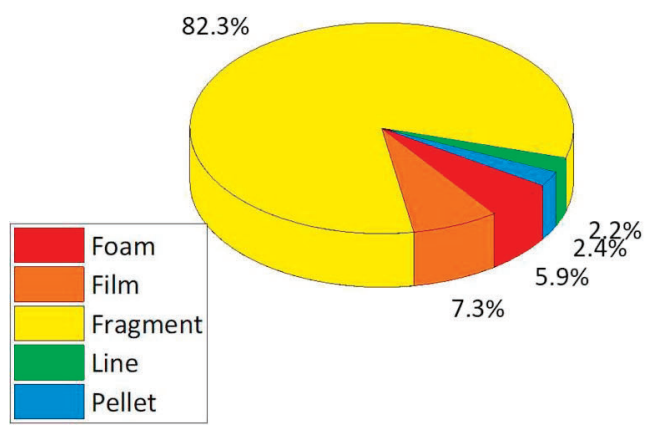

Mesoplastics

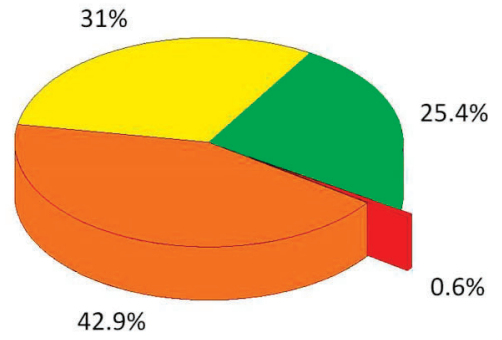

Fig. 3. The ratios for the shape of particles whose size range falls within the micro (left) and meso (right) size ranges. The color legend for both charts is present in the lower left corner. A more detailed size breakdown for each shape category can be seen in Fig. SI2. (For interpretation of the references to color in this figure legend, the reader is referred to the web version of this article.)

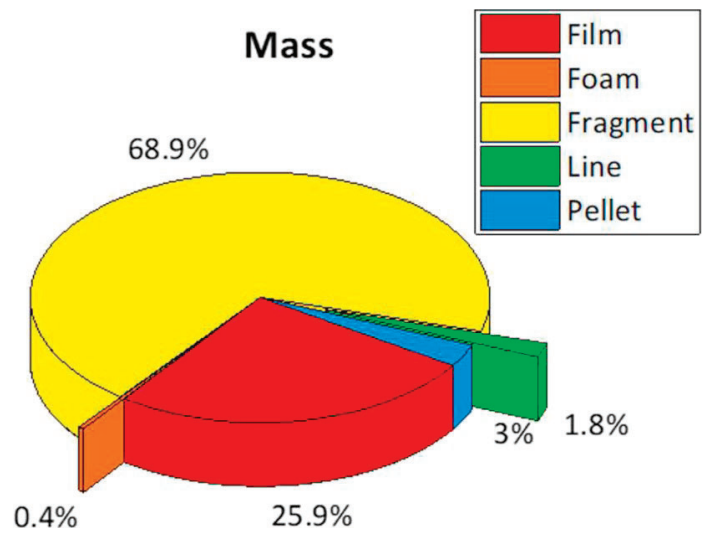

Fig. 4. The final ratios of particle shape, calculated according to particle mass, for the overall sample.

in Fig. 5, was used as a guide for the selection of representative particles. A slight modification was made to this initial ratio to ensure each category had a minimum of 10 representative particles. The particles were further sorted manually according to their size.

ATR FT-IR spectra collected from the representative particles showed that the two dominant plastic types represented in the sample, as shown in Fig. 6, were polyethylene and polypropylene. In addition to these two plastic types, polyamide and polystyrene were also highly prevalent. Additional polymer types (i.e. poly(1-butene), polymethylepentene, nylon 12, and poly(4-methyl-1-pentene)) were detected at very low amounts and were sorted into a category titled 'other' to aid the ease of reading for the charts. By considering the predominant plastic types for each of the particle shape categories, it can be seen that polyethylene and polypropylene are the main plastic types for all categories other than foams. Nearly all foam samples were composed of expanded polystyrene.

Matches between the representative particles and the pristine polymer spectra can be seen in Fig. 7 .

\section{Discussion}

Plastic pollution appears to be ubiquitous within the Ligurian and Tyrrhenian Seas. While the concentration of plastic particles differed among samples, every sample taken contained plastics. The majority of our sample was composed of microplastic particles $(\sim 65 \%)$, with a smaller portion being comprised of mesoplastics ( $\sim 35 \%)$. Having the majority of the sample be within the microplastic size range is a finding which stands in agreement with studies conducted in seas and oceans across the globe, such as the study published by Eriksen et al. (2014) that reported microplastic particles composed the majority of their samples and may account for as much as $92 \%$ of the global plastic particle count in oceans (Eriksen et al., 2014). However, the concentration of plastic particles reported in this study $(28,376 \pm 28,917$ particles $\mathrm{km}^{-2}$ ) is lower than those reported for similar studies in the area, such as the one conducted by Baini et al. that reported an average of $69,161 \pm 83,244$ particles $\mathrm{km}^{-2}$ (Baini et al., 2018). One plausible explanation for such differences is the high heterogeneity of the plastic pollution present within the Mediterranean, which has been referred to
A

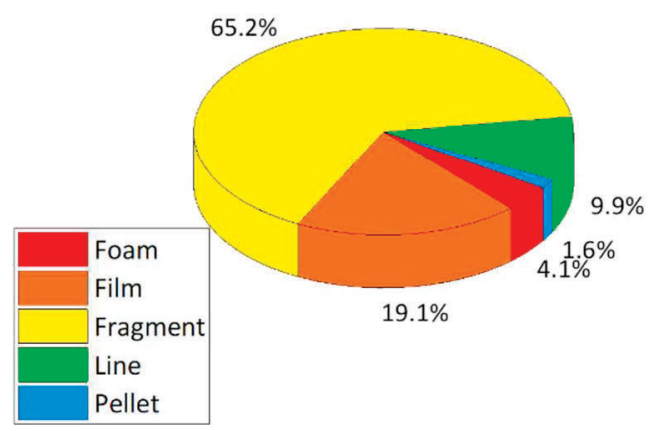

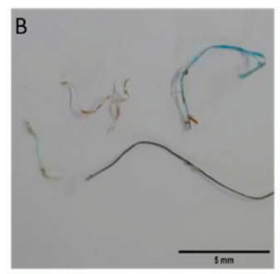
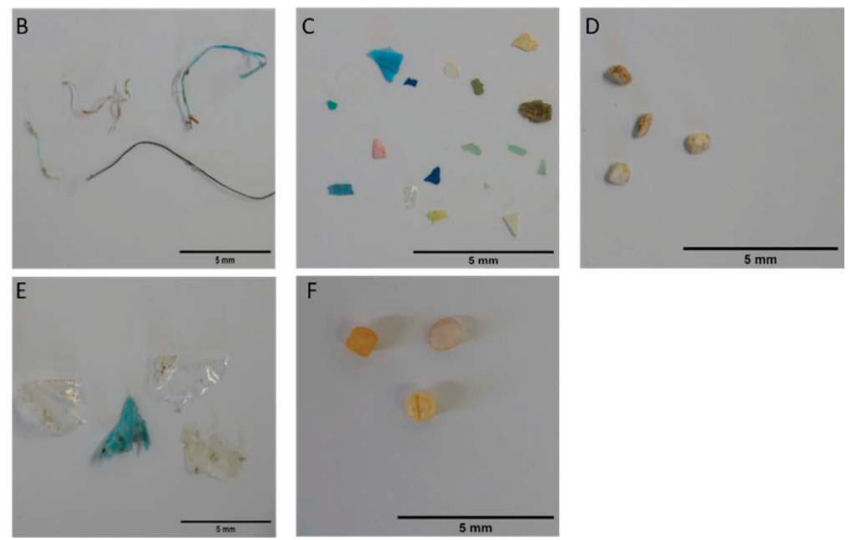

Fig. 5. The final ratios of particle shape for the overall sample (A) and representative images of the particles from each shape category. The categories include lines (B), fragments (C), foams (D), films (E), and pellets (F). Scale bars: $5 \mathrm{~mm}$. 
Whole Sample

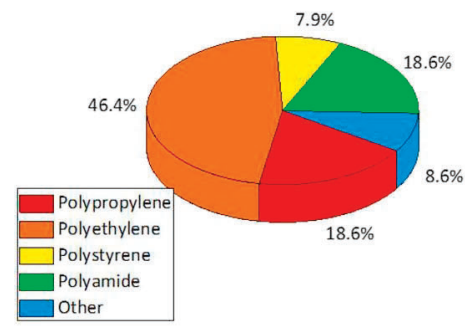

Foam

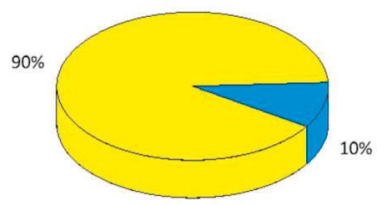

Film

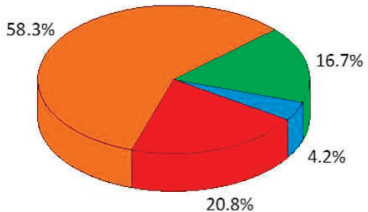

Line

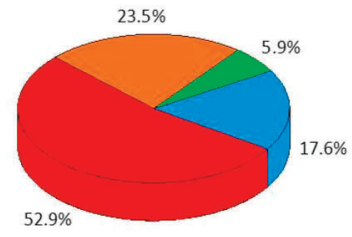

Fragment

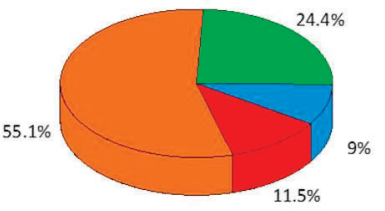

Pellet

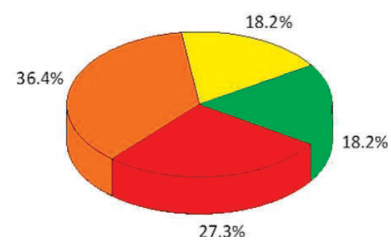

Fig. 6. The polymer composition of the entire sample of representative particles (top left) and the polymer composition for the particles sorted according to their shape. Data was obtained for a representative sample of 140 particles (78 fragments, 24 films, 17 lines, 11 pellets, 10 foams). The color legend used for all pie charts in the figure is displayed in lower left corner of the whole sample box. (For interpretation of the references to color in this figure legend, the reader is referred to the web version of this article.)
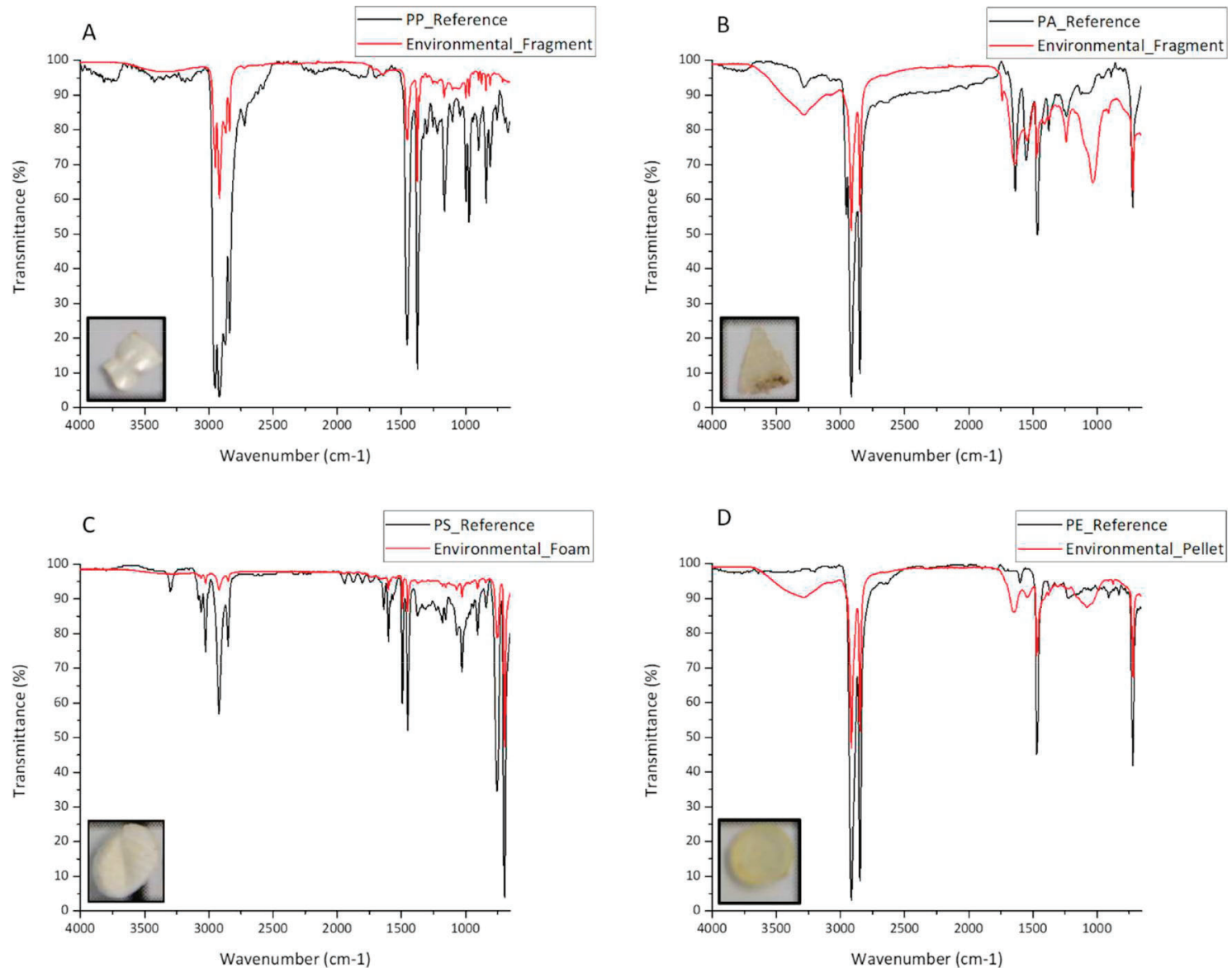

Fig. 7. A comparison of pristine polymer spectra (black) with spectra collected from representative particles from the sample (red). The polypropylene reference is compared to the spectra of a representative fragment (A). The polyamide reference is compared to the spectra of an additional representative fragment (B). The polystyrene reference is compared to the spectra of representative foam (C). The polyethylene reference spectrum is compared to a representative pellet sample (D). Representative particles are shown as insets in the lower left corner of their spectra. (For interpretation of the references to color in this figure legend, the reader is referred to the web version of this article.) 
as a 'plastic soup' in the literature (Suaria et al., 2016). This high heterogeneity leads some researchers, such as Guerrini et al. (2019), to argue that environmental sampling campaigns within the region provide only a temporally specific snapshot of the state of the Mediterranean (Guerrini et al., 2019). Thus, differences in the concentrations obtained at different times, particularly if the samples are collected years apart, are likely to be the result of the region's heterogenous pollution distribution. Yet another potential explanation for such a discrepancy in numbers could be the lack of a digestion protocol for organic matter and subsequent re-analysis of the samples in this study.

When considering the sample according to the shape of the particles, fragments account for the bulk of the overall sample collected (65\%) with the fraction of the microplastic sample that is fragments being even higher (82\%). Studies conducted by Baini et al. (2018) and Gündoğdu and Çevik (2017) have also reported the largest fraction of their samples were plastic fragments ( $81 \%$ and $\sim 60 \%$, respectively) (Baini et al., 2018; Gündoğdu and Çevik, 2017). This high prevalence of microplastic fragments is likely the result of weathering of macro- and mesoplastics through UV light and mechanical force from wind and waves.

Natural weathering along with chemical modifications during the processing such as the addition of dyes, copolymers, plasticizers, or other common plastic additives can impact further chemical analysis by FT-IR (Barbes et al., 2014). Despite the difficulties this may pose for later analysis and interpretation, it is important to attempt to confirm - the chemical identity of the particles sampled to ensure particles composed of biopolymers (i.e. chitin, lignin, cellulose) are not counted as pollutants. The result of chemical analysis revealed that the predominant polymer types in our samples (i.e. for meso and microplastics) were polyethylene (46\%), polypropylene (19\%), polystyrene $(8 \%)$, and polyamide (19\%). These polymer types are reported to be among the most common types utilized in the production of food packaging, plastic bags, children's toys, and other common consumer goods, and were reported as the most prevalent in additional studies conducted in the area (PlasticsEurope, 2018; Baini et al., 2018).

All studies which sample plastic from the environment report high heterogeneity in the number of particles obtained from each sampling site (Güvena et al., 2017; Gündoğdu and Çevik, 2017). This pattern is continued in our study, with the final average particle count of 28,376 in one square kilometer having a standard deviation of 28,917 . This high standard deviation can be easily understood when one considers the range of particle abundance determined at each sampling site, with a minimum abundance of 1,009 particles $\mathrm{km}^{-2}$ and a maximum of 122,817 particles $\mathrm{km}^{-2}$ found.

Understanding the source of this high variation in particle abundance requires one to consider many factors such as the proximity of the sampling location to busy harbors (e.g. Port of Genoa), industrial zones, or large rivers. Within the region sampled during this study, the Magra, Serchio, Arno, Ombrone, Tevere, Tavignano, Golo, Tirso, and Flumendosa rivers flow directly into the sea. Additionally, the study by Guerrini et al. points out the importance of accounting for the potential impact of multiple major marine shipping routes, such as those running from Genoa or Livorno to Barcelona (Guerrini et al., 2019). However, as only buoyant materials were collected during this study, it is very important to consider the overall influence of ocean currents on the movement of the particles. Thus, if a sample is taken near a busy harbor, but the current is strong in the region, it is possible that relatively little pollution will be observed. In areas where the currents are weak, or where they move in a cyclonic fashion, it will be more likely to see the accumulation of plastic particles. These currents are driven by many factors such as the temperature of the water and the topography of the ocean floor and surrounding landmasses.

Fossi et al. have attempted to utilize satellite data for the Mediterranean region to model the potential distribution of microplastic particles (Fossi et al., 2017). These models predict accumulation of the particles in the Ligurian and Tyrrhenian Seas will occur predominantly in the Capria Gyre, which is located between the eastern coast of Corsica and the coast of Livorno, and the Northern Tyrrhenian Gyre, also located off the eastern coast of Corsica, but closer to the northern coast of Sardinia (i.e. located further south than the Capria Gyre). Comparing the map of sample locations and particle densities for this study shown in Fig. 1 with the predicted accumulation zones shown in the simulations created by Fossi et al. (2017) shows a similar pattern. Sample 5 (shown labelled in Fig. SI1), collected off the coast of Livorno (i.e. near the Capria Gyre), shows a high spike in particle density $\left(68,457\right.$ particles $\mathrm{km}^{-2}$ (shown in Table SI1)) compared to sample 4 $\left(3,665\right.$ particles $\left.\mathrm{km}^{-2}\right)$ which was collected slightly north of Livorno. Samples 6 to 34, taken between the area near Livorno and the eastern coast of Corsica (i.e. near the Capria and Northern Tyrrhenian Gyres), also had relatively high particle densities when compared with the northernmost samples collected.

Samples were collected in two-week increments differentiated by the direction of travel. Samples 1 to 19 were collected as the ship sailed south, while samples 20 to 34 were collected as the ship sailed northward. While some sample sites, such as the site for sample 7 and 29 (Fig. SI1), were very close to each other geographically, different particle concentrations were estimated. Samples 7 and 29, collected approximately 1.5 weeks apart, had concentrations of 24,013 particles $\mathrm{km}^{-2}$ and 122,817 particles $\mathrm{km}^{-2}$, respectively. Samples 18 and 20, which were collected approximately 1.5 days apart, also showed a slight variation in the total particle concentration observed $(16,128$ particles $\mathrm{km}^{-2}$ and 11,536 particles $\mathrm{km}^{-2}$, respectively). Such variation within a sample location may be caused by natural phenomena such as the effect of wind speed and direction on the sea surface roughness, or could be caused by the direction the ship sails compared to the direction of the ocean current (i.e. sailing with or against the movement of the water). Thus, it becomes apparent that high variation is inherent in samples collected from a dynamic environment such as the Mediterranean Sea.

With high variability being an inherent trait of environmental samples due to natural phenomena, thus creating a 'plastic soup' for which only temporal snapshots can be obtained, it is important for researchers to begin working towards a standardized procedure for sample collection and processing. To be able to make direct comparisons between studies, it is important to ensure that factors such as net type and mesh size, sieve or filtration protocols, and sorting categories are the same. Thus, this study aims to lay the foundation for future sampling experiments which would allow for a better understanding of the long-term presence and behavior of plastic particles in the region. Small changes, such as the season the samples are collected, could then be made in order to conduct further experiments which give more understanding of the impact of various potential plastic particle sources. For example, as this region is a hotspot for tourism, with nearly 200 million tourists visiting the region within a year, the influx of plastic from beaches is a key source of plastic pollution within the Mediterranean, and is one which is highly variable; increasing pollution levels as much as $40 \%$ during the summer season (Galgani, 2014). Thus, it could also be of interest to use such standardized testing methods to collect samples from within the region during various seasons (e.g. the high and low seasons for tourism) over the course of several years to aid in the understanding of the impact of such variable pollution sources. Additionally, an experimental setup similar to the transect sampling conducted by Baini et al. could be conducted near regions where majors rivers flow into the Mediterranean, or near busy harbors such as the Port of Genoa, in order to provide a better understanding of the true impact of these potential pollution sources, and the long-term fate of the plastic particles introduced into the Mediterranean via such sources (Baini et al., 2018).

\section{Conclusions}

Microplastic particles were found in all samples collected within the 
Ligurian and Tyrrhenian Seas during this study. The most common polymer types in these samples were identified with ATR FT-IR and aligned well with the most common polymers produced and sold commercially, i.e. polyethylene, polypropylene, polystyrene, and polyamide. A high variability in the abundance of particles present in the samples collected, which spanned 1,009 to 122,817 particles $\mathrm{km}^{-2}$ could be shown. This high variability among the samples is likely the result of variations in the Seas' currents and weather. Most samples collected near the Capria and Northern Tyrrhenian Gyres had significantly higher particle densities than samples collected from waters further north.

All samples in this study were collected from surface water with a net mesh of $333 \mu \mathrm{m}$. Monitoring changes in the particle densities over time could be achieved by revisiting the same sites and ensuring sample collection is conducted in the same manner. These repeated collections would allow for insight into whether the pollution levels within the region are increasing over time and could potentially be used to track the effectiveness of any future regulations imposed on plastic use or disposal. In addition to studies of surface waters, it will be necessary to conduct experiments which investigate samples collected from various depths within the Seas and their sediment. Ingestion of these materials by various aquatic organisms in the region (i.e. fish, mussels) could then be studied with the potential to compare the effects of the pollution in accumulation zones with regions of lower particle concentrations. These samples would help to give a full representation of the distribution and fate of plastic waste in marine environments.

\section{Acknowledgements}

The authors would like to thank all the crew members of the 2018 Expedition conducted by the Sail \& Explore Association for supporting - sample collection as well as the team of the Hydra marine field station on Elba. The authors would additionally like to thank the Adolphe Merkle Foundation for their support during subsequent sample analysis.

\section{Declaration of competing interest}

The authors have no conflicts of interest to report.

\section{Appendix A. Supplementary data}

Supplementary data to this article can be found online at https:// doi.org/10.1016/j.marpolbul.2019.110572.

\section{References}

Baini, M., Fossi, M.C., Galli, M., Caliani, I., Campani, T., Grazia Finoia, M., Pantia, C., 2018. Abundance and characterization of microplastics in the coastal waters of Tuscany (Italy): the application of the MSFD monitoring protocol in the Mediterranean Sea. Mar. Pollut. Bull. 133, 543-552.

Barbes, L., Radulescu, C., Stihi, C., 2014. ATR-FTIR spectrometry characterisation of polymeric materials. Romanian Rep. Phys. 66 (3), 765-777 2014.

Brennecke, D., Duarte, B., Paiva, F., Caçador, I., Canning-Clodeade, J., 2016. Microplastics as vector for heavy metal contamination from the marine environment. Estuar. Coast. Shelf Sci. 178, 189-195.

Browne, M.A., Crump, P., Niven, S.J., Teuten, E., Tonkin, A., Galloway, T., Thompson, R., 2011. Accumulation of microplastic on shorelines worldwide: sources and sinks. Environ. Sci. Technol. 45 (21), 9175-9179.

Moore, C.J., 2008. Synthetic polymers in the marine environment: a rapidly increasing, long-term threat. Environ. Res. 108 (2), 131-139.

Collignon, A., Hecq, J., Glagani, F., Voisin, P., Collard, F., Goffart, A., 2012. Neustonic microplastic and zooplankton in the North Western Mediterranean Sea. Mar. Pollut Bull. 64 (4), 861-864.

Connors, P.G., Smith, K.G., 1982. Oceanic plastic particle pollution: suspected effect on fat deposition in red phalaropes. Mar. Pollut. Bull. 13 (1), 18-20.
Galgani, F, 2014Distribution, composition and abundance of marine litter in. Distribution, composition and abundance of marine litter in the Mediterranean and Black Seas. Monaco: s.n. In: CIESM Workshop Monograph, pp. 180.

Eriksen, M., Lebreton, L.C.M., Carson, H.S., Thiel, M., Moore, C.J., Borerro, J.C., Galgani, F., Ryan, P.G., Reisser, J., 2014. Plastic pollution in the world's oceans: more than 5 trillion plastic pieces weighing over 250,000 tons afloat at sea. 12. PLoS One 9, e111913.

Faure, F., Saini, C., Potter, G., Galgani, F., de Alencastro, L.F., Hagmann, P., 2015. An evaluation of surface micro- and mesoplastic pollution in pelagic ecosystems of the Western Mediterranean Sea. Environ. Sci. Pollut. Res. 22 (16), 12190-12197.

Fossi, M.C., Marsili, L., Baini, M., Giannetti, M., Coppola, D., Guerranti, C., Caliani, I., Minutoli, R., Lauriano, G., Finoia, M.G., Rubegni, F., Panigada, S., Bérubé, M., Ramírez, J.U., Panti, C., 2016. Fin whales and microplastics: the Mediterranean Sea and the Sea of Cortez scenarios. Environ. Pollut. 209, 68-78.

Fossi, M.C., Romeo, T., Baini, M., Panti, C., Marsili, L., Campani, T., Canese, S., Galgani, F., Druon, J., Airoldi, S., Taddei, S., Fattorini, M., Brandini, C., Lapucci, C., 2017. Plastic debris occurrence, convergence areas and fin whales feeding ground in the Mediterranean marine protected area Pelagos Sanctuary: a modeling approach. Front. Mar. Sci. 4.

Galgani, F., Leaute, J.P., Moguedet, P., Souplet, A., Verin, Y., Carpentier, A., Goraguer, H., Latrouite, D., Andral, B., Cadiou, Y., Mahe, J.C., Poulard, J.C., Nerisson, P., 2000. Litter on the sea floor along European coasts. Mar. Pollut. Bull. 40 (6), 516-527.

Guerrini, F., Mari, L., Casagrandi, R., 2019. Modeling plastics exposure for the marine biota: risk maps for fin whales in the Pelagos Sanctuary (North-Western Mediterranean). Front. Mar. Sci. 6, 299.

Gündoğdu, S., 2017. High level of micro-plastic pollution in the Iskenderun Bay NE Levantine coast of Turkey. J. Fish. Aquat. Sci. 34 (4), 401-408.

Gündoğdu, S., Cevik, C., 2017. Micro- and mesoplastics in Northeast Levantine coast of Turkey: the preliminary results from surface samples. 1-2. Mar. Pollut. Bull. 118, 341-347.

Güvena, O., Gökdağa, K., Jovanović, B., Kıdey, A.E., 2017. Microplastic litter composition of the Turkish territorial waters of the Mediterranean Sea, and its occurrence in the gastrointestinal tract of fish. Environ. Pollut. 223, 286-294.

van der Hal, N., Ariel, A., Angel, D.L., 2017. Exceptionally high abundances of microplastics in the oligotrophic Israeli Mediterranean coastal waters. Mar. Pollut. Bull. 116 (1-2), 151-155.

Jambeck, J.R., Geyer, R., Wilcox, C., Siegler, T.R., Perryman, M., Andrady, A., Narayan, R., Lavender Law, K., 2015. Plastic waste inputs from land into the ocean. 6223. Science 327, 768-771.

Lebreton, L., Slat, B., Ferrari, F., Sainte-Rose, B., Aitken, J., Marthouse, R., Hajbane, S., Cunsolo, S., Schwarz, A., Levivier, A., Noble, K., Debeljak, P., Maral, H., SchoeneichArgent, R., Brambini, R., Reisser, J., 2018. Evidence that the Great Pacific Garbage Patch is rapidly accumulating plastic. Sci. Rep. 8 (1).

Lusher, A.L., Tirelli, V., O'Connor, I., Officer, R., 2015. Microplastics in Arctic polar waters: the first reported values of particles in surface and sub-surface samples. Sci. Rep. 5.

von Moos, N., Burkhardt-Holm, P., Köhler, A., 2012. Uptake and effects of microplastics on cells and tissue of the blue mussel Mytilus edulis L. after an experimental exposure. Environ. Sci. Technol. 46 (20), 11327-11335.

Center for International Earth Science Information Network (CIESIN) (Ed.), 2012. National Aggregates of Geospatial Data Collection: Population, Landscape, and Climate Estimates Version 3 (PLACE III).

Pedrotti, M.L., Petit, S., Elineau, A., Bruzaud, S., Crebassa, J., Dumontet, B., Martí, E., Gorsky, G., Cózar, A., 2016. Changes in the floating plastic pollution of the Mediterranean Sea in relation to the distance to land. PLoS One 11 (8), e0161581.

PlasticsEurope, 2017. Plastics - The Facts 2017 - An Analysis of European Plastics Production, Demand and Waste Data. PlasticsEurope.

PlasticsEurope, 2018. Plastics - The Facts 2018. PlasticsEurope, Brussels.

Rochman, C., Hoh, E., Kurobe, T., Teh, S., 2013. Ingested plastic transfers hazardous chemicals to fish and induces hepatic stress. Sci. Rep. 3, 3263.

Ruiz-Orejón, L.F., Sardá, R., Ramis-Pujol, J., 2016. Floating plastic debris in the Central and Western Mediterranean Sea. Mar. Environ. Res. 120, 136-144.

Ruiz-Orejón, L.F., Sardá, R., Ramis-Pujol, J., 2018. Now, you see me: high concentrations of floating plastic debris in the coastal waters of the Balearic Islands (Spain). Mar. Pollut. Bull. 133, 636-646.

van Sebille, E., Wilcox, C., Lebreton, L., Maximenko, N., Hardesty, B.D., van Franeker, J.A., Eriksen, M., Siegel, D., Galgani, F., Lavender Law, K., 2015. A global inventory of small floating plastic debris. Environ. Res. Lett. 10, 124006.

Suaria, G., Mineo, A., Lattin, G.L., Magaldi, M.G., Belmonte, G., Moore, C.J., Regoli, F., Aliani, S., 2016. The Mediterranean Plastic Soup: synthetic polymers in Mediterranean surface waters. Sci. Rep. 6, 37551.

Van Cauwenberghe, L., Vanreusel, A., Mees, J., Janssen, C.R., 2013. Microplastic pollution in deep-sea sediments. Environ. Pollut. 182, 495-499.

Watts, A.J.R., Urbina, M.A., Corr, S., Lewis, C., Galloway, T.S., 2015. Ingestion of plastic microfibers by the crab Carcinus maenas and its effect on food consumption and energy balance. Environ. Sci. Technol. 49 (24), 14597-14604.

Wright, S.L., Kelly, F.J., 2017. Plastic and human health: a micro issue? Environ. Sci. Technol. 51 (12), 6634-6647. 